\title{
Risultati delle osservazioni ionosferiche effettuate in Roma durante l'eclissi solare del 15 Febbraio 1961
}

\author{
A. Bolle - F. Coccia - C. A. Tirerio $(*)$
}

Ricevuto il 5 Giugno 1961

Lo scopo di questa comunicazione è di rappresentare alcuni risultati delle osservazioni ionosferiche effettuate in Roma in occasione dell'eclisse di sole del 15 Febbraio 1961, che permettono di valutare la maggiore superficie del sole ionizzante rispetto al sole ottico.

Le misure sono state eseguite presso l'Osservatorio ionosferico " $\mathrm{S}$. Alessio " dell'Istituto Nazionale di Geofisica in collaborazione con il Comitato Telecomunicazioni Militari.

Il sondaggio ionosferico è stato effettuato nella suddetta stazione perchè alla latitudine di Roma corrispondeva la totalità dell'eclisse alla quota di $100 \mathrm{~km}$ e quindi per lo strato $E$.

L'apparechiatura è quella ordinariamente impiegata per il rilevamento sistematico delle caratteristiche ionosferiche, col metodo del sondaggio ad impulsi con incidenza verticale e consta di una ionosonda di costruzione inglese Union Radio tipo MIKII e di un sistema di quattro antenne rombiche verticali, due per la emissione e due per la ricezione.

La gamma di frequenza esplorata va da $0,6 \mathrm{MHz}$ a $25 \mathrm{MHz}$, divisa in 5 sottogamme. Le marche di calibrazione in frequenza ed in altezza sono ottenute per divisione di frequenza da un oscillatore controllato a quarzo. La potenza di picco è compresa tra $1,8 \mathrm{kw}$ e $0,5 \mathrm{kw}$, rispettivamente all'estremo inferiore e superiore della gamma di frequenza.

In aggiunta alla sonda panoramica descritta è stata messa a punto, a scopo di controllo, un'altra sonda a frequenza fissa lavorante a $2,4 \mathrm{MHz}$, con funzionamento continuo. Sono state altresì effettuate misure relative di campo su varie emittenti nella gamma delle onde corte e medie.

In occasione dell'eclisse il programma dei sondaggi, che d'ordinario prevede una cadenza di $30^{\mathrm{m}}$ è stata intensificata in modo da ottenere

(*) Istituto Nazionalo di Geofisica - Roma. 


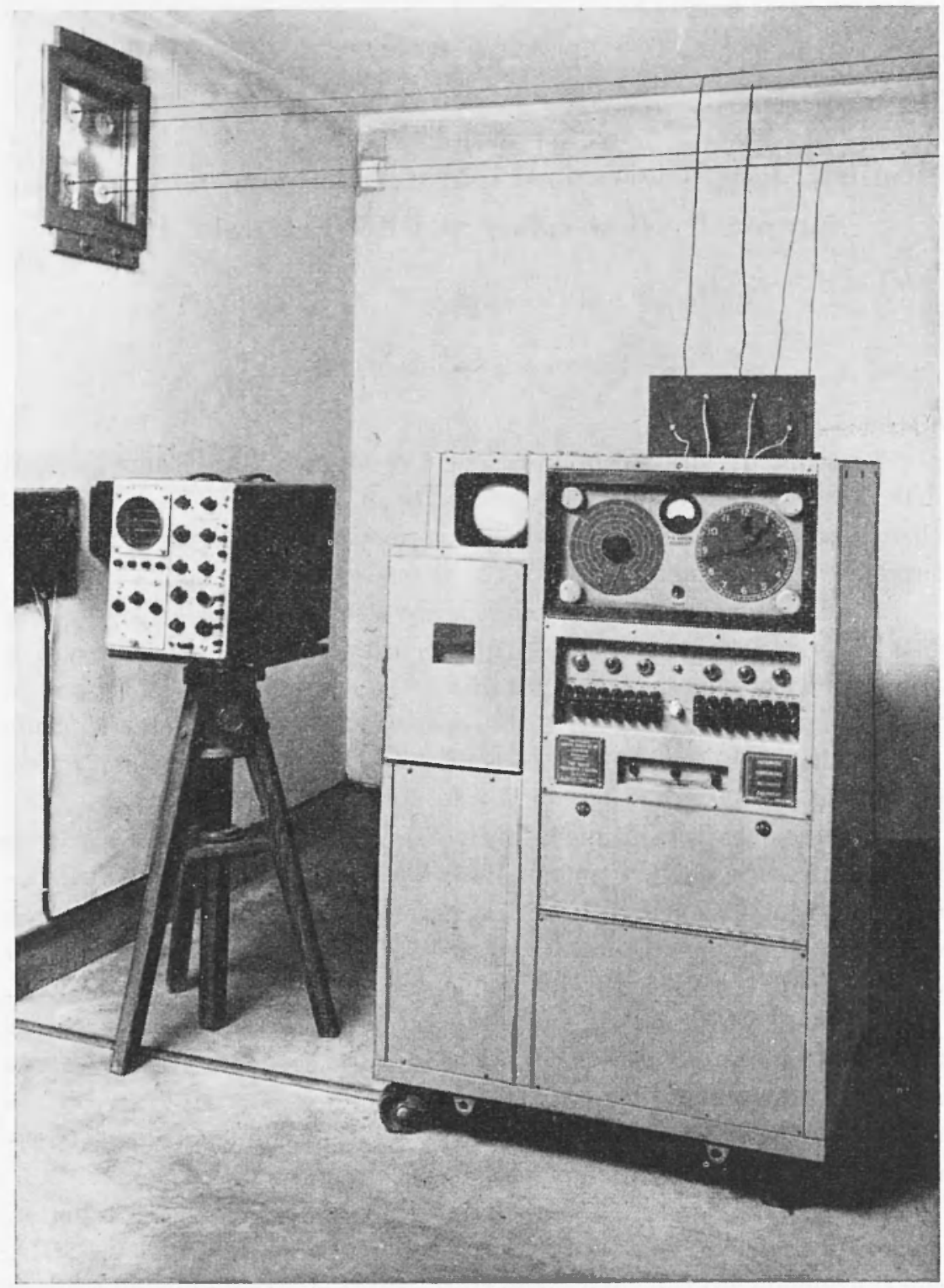

Fig. 1. - Sonda ionosferica tipo MK 2.

il massimo numero di ionogrammi compatibili con il ciclo automatico di funzionamento dell'apparato. In aggiunta alla registrazione fotografica del tempo sugli ionogrammi, l'ora di inizio di esplorazione di ogni 
sottoganma è stata controllata separatamente per confronto con un cronometro di precisione, in modo da poter contare su un approssimazione di \pm 1 secondo.

Sulla verticale di Roma ed alla quota di $100 \mathrm{~km}$ l'eclisse è stata totale alla ore $08^{\mathrm{n}} 34^{\mathrm{m}} 20^{\mathrm{s}}$ T.M.E.C.

Nella Fig. 1 è mostrato l'andamento dell'eclisse in quota. Là carta è stata compilata sulla scorta dei dati astronomici gentilmente forniti dal Prof. A. Kranje che ci è gradito ringraziare in questa occasione.

Nella Fig. 3 è mostrato l'f-plot del giorno 1o, unitamente alla curva mediana mensile di Febbraio.

Nelle Figg. 2 e 4 sono riportate per confronto gli f-plots dei giorni 14 e 16.

Una presentazione più dettagliata dell'andamento delle frequenze critiche nelle ore precedenti e seguenti l'eclisse viene data in Fig. 5, che riporta oltre le frequenze critiche il corrispondente valore delle densità elettroniche.

Da tale figura si rileva che il minimo di ionizzazione nella regione F2 è intervenuto alle ore 08.39 , con un ritardo di sette minuti circa rispetto alla fase massima ottica al suolo. La normalita sia pure con valori inferiori alla mediana si è ristabilita a partire dalle ore 10 .

Le osservazioni della frequenza critica dello strato $E$ risultano incomplete, specie in corrispondenza dei valori più bassi che renivano a cadere nella banda di frequenza delle stazioni di radio diffusione. Lo studio degli ionogrammi sembra tuttavia escludere la totale scomparsa della ionizzazione.

Le misure di intensità di campo della stazione di Radio Palermo eseguite alla Sede Centrale dell'Istituto di Geofisica denotano un brusco abbassamento al momento della formazione dello strato $E$ ed un ammento dell'intensità di campo durante l'eclisse con massimo piatto lievemente precedente la fase di totalità.

Lo studio degli ionogrammi dello strato $F$ mostra la presenza delle stratificazioni $F 1$ ed $F^{2}$ durante l'eclisse, nonchè un rilevante numero di echi multipli imputabili a scarso assorbimento negli strati più bassi.

$$
\frac{d N}{d t}=q(t)-\alpha N^{2}
$$

in cui $N$ è il numero di elettroni $\left(\mathrm{cm}^{-3}\right), q(t)$ il tasso di produzione di elettroni $\left(\mathrm{cm}^{-3} \mathrm{e} \mathrm{sec}^{-1}\right)$, ed a il coefficiente di ricombinazione $\left(\mathrm{cm}^{-3} \mathrm{sec}^{-1}\right)$.

Non si è tenuto conto del coefficiente di attaccamento perchè da ritenersi trascurabile per la regione $F 2$. 


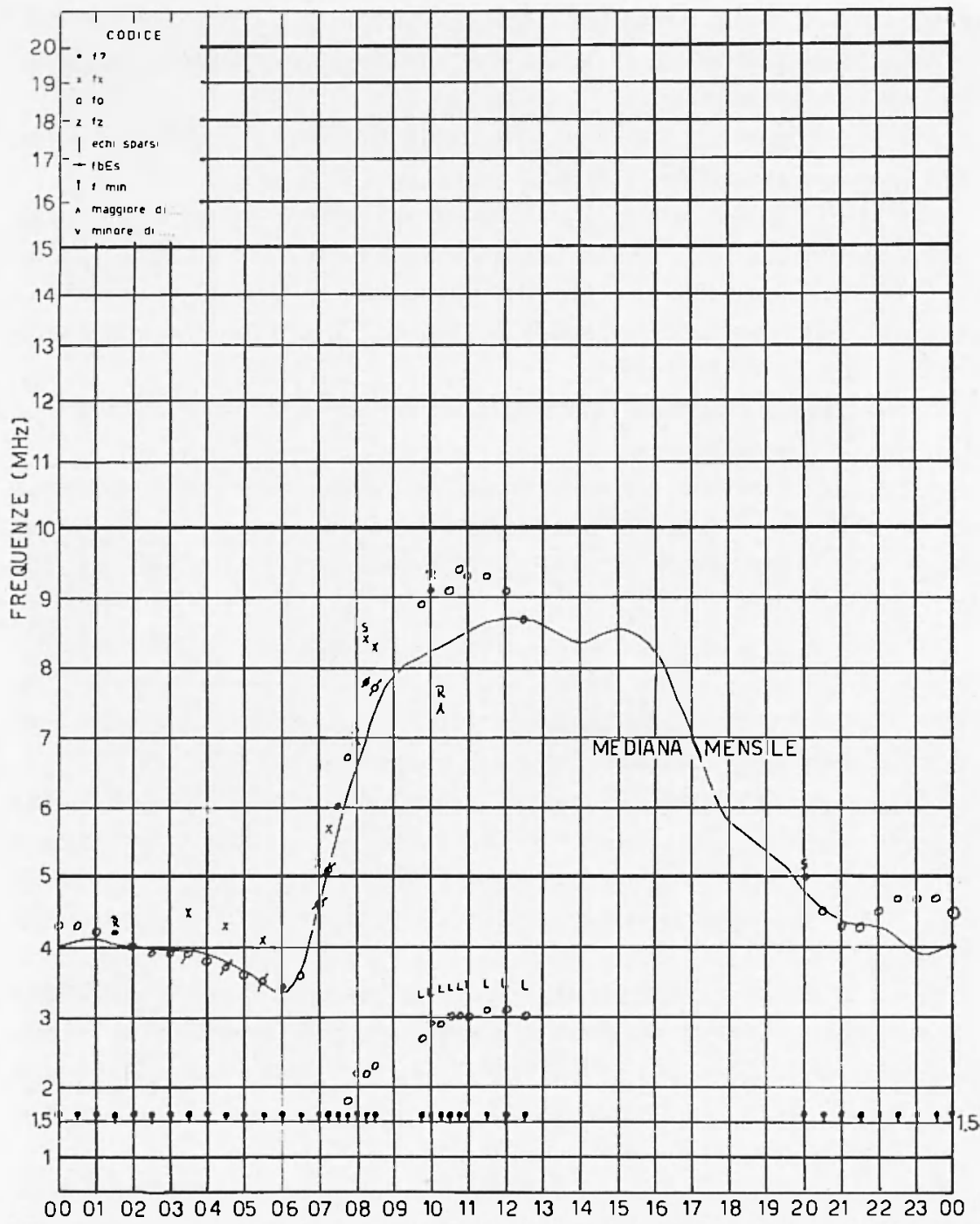

Fig. 2. - Diagramma delle frequenze (f-plot) del giomo 14-2-1961.

In tale equazione si ì posto $q=0$ in corrispondenza dell'istante di fase massima ottenendo per a un valore di circa $7 \times 10^{-10}$, molto diverso con il valore $40 \times 10^{-10}$ commemente accettato per la quota di $220 \mathrm{~km}$.

Ciò porta a ritenere che anche nelle condizioni di massima totalità la superficie ionizzante solare non sia totalmente occultata 0 , in altri 


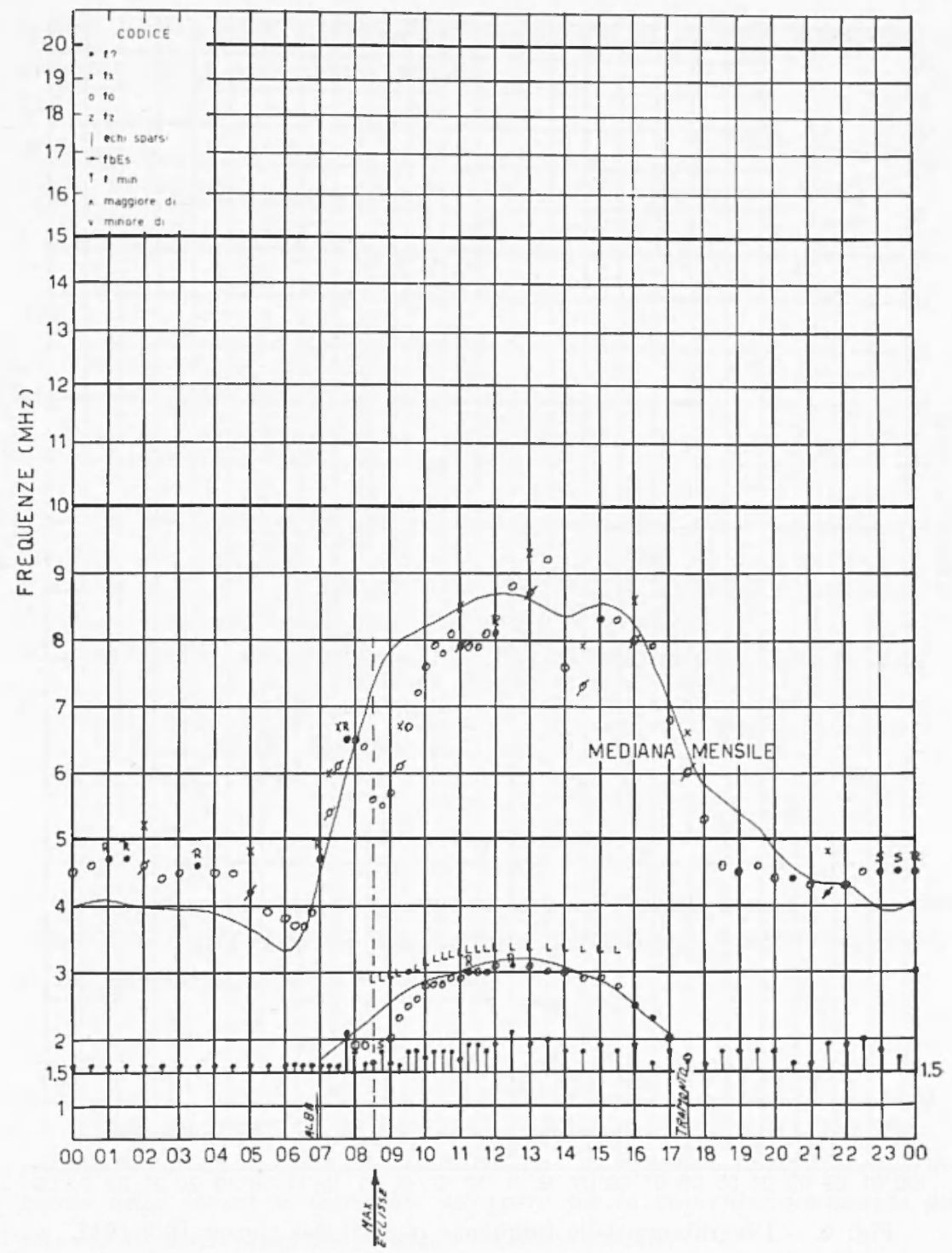

Fig. 3. - Diagramma delle frequenze (f-plot) del giomo 15-2-1961.

termini, che agli efletti ionosferici l'eclisse si presenti come anulare anzichè totale.

Si è pensato cosi di calcolare il rapporto tra la superficie ionizzante solare e quella del disco visibile ritenuto, in prima approssimazione eguale alla superficie occultata attribuendo ad $a$ il valore $40 \times 10^{-10}$ per la quota di $220 \mathrm{~km}$. 


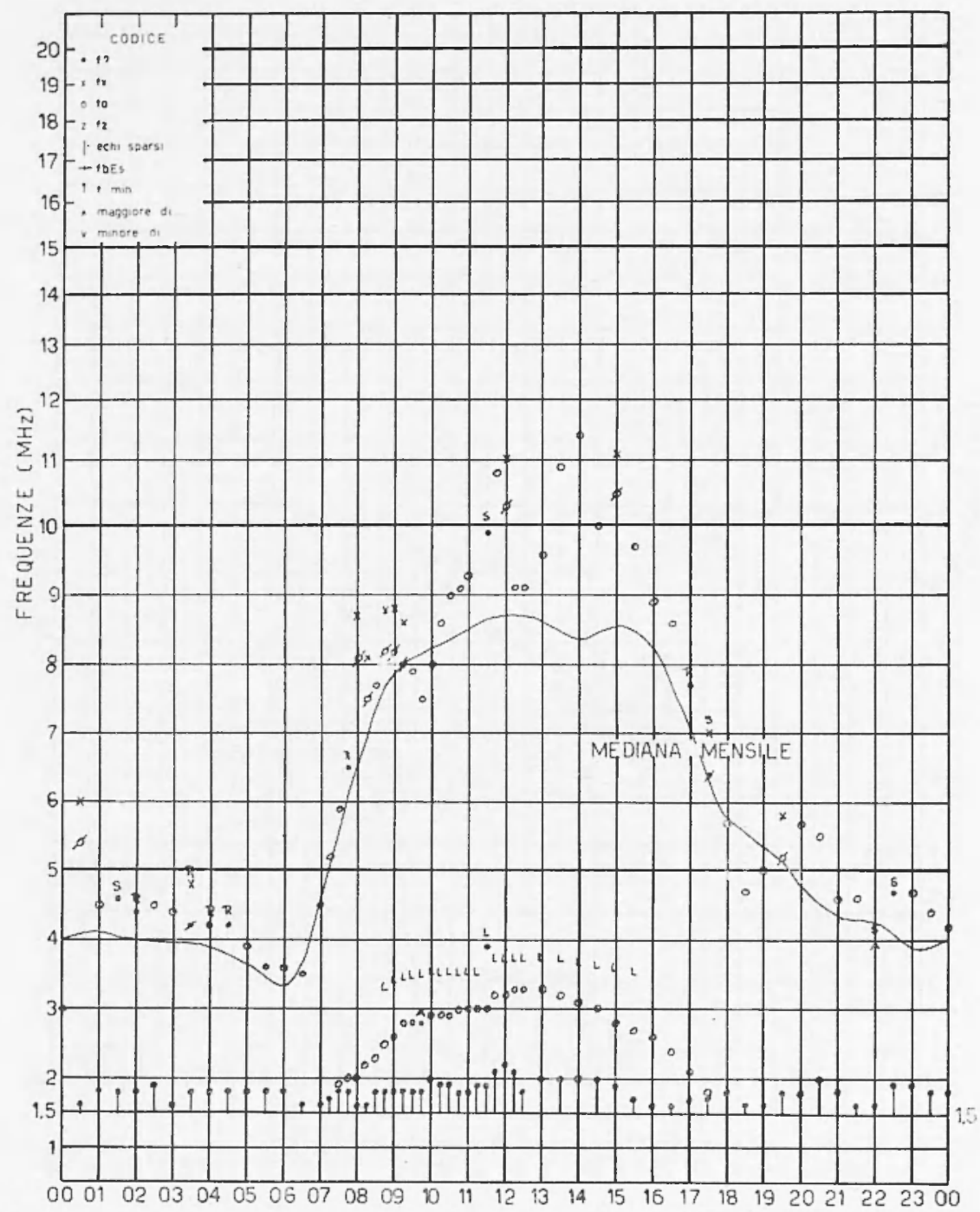

Fig. 4. - Diagramma delle frequenze (f-plot) del giorno 16-2-1911.

Si è trovato per $q$ il valore di 450 elettroni $\mathrm{cm}^{-3} \mathrm{sec}^{-1}$.

Confrontando questo valore con quello dedotto dalla curva mediana per lo stesso tempo, che risulta di circa 2000 elettroni $\mathrm{cm}^{-3} \mathrm{sec}^{-1}$ si è trovato per il rapporto predetto il valore di 1,25 .

Purtroppo la corrispondente diminuzione di frequenza critica per lo strato $E$, la stazionarietà che ne deriva, di circa $14^{\mathrm{m}}$ della frequenza 
critica, è solo poco chiaramente rilevabile dagli ionogrammi in nostro possesso per la suaccennata ragione.

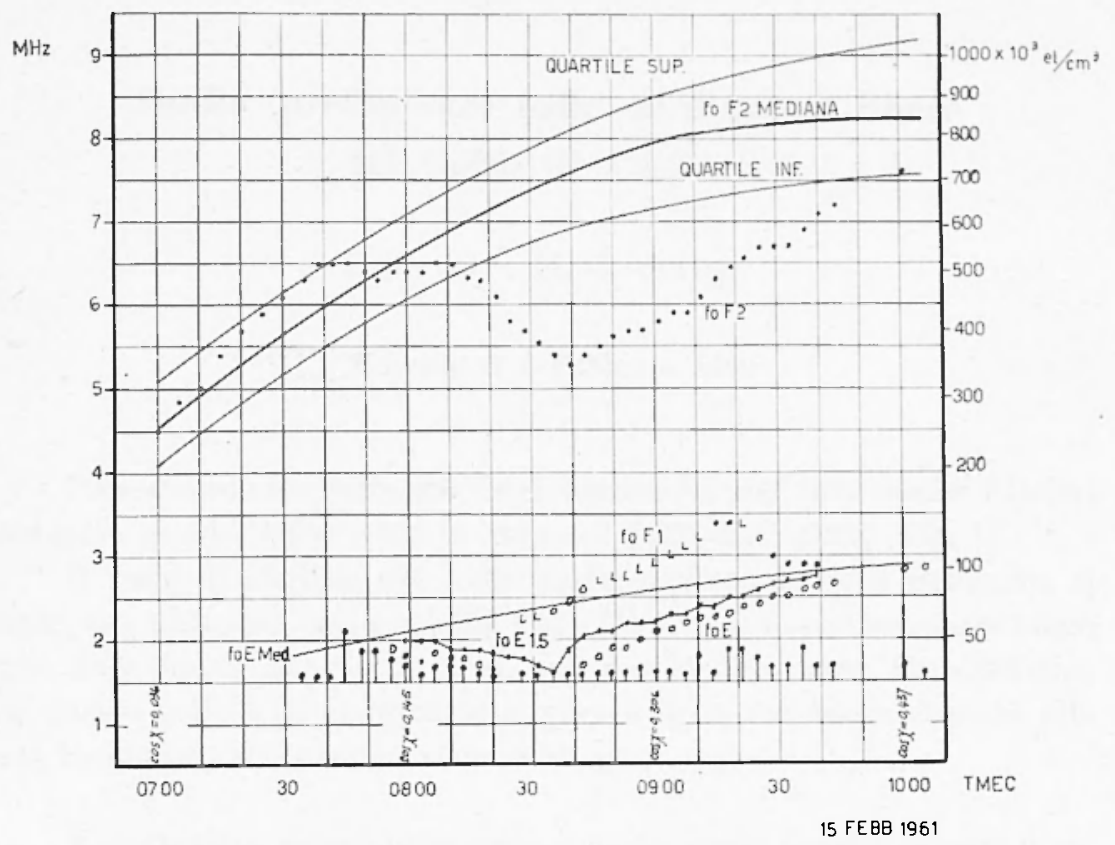

Fig. 5.

Migliore conferma di quanto esposto ci ripromettiamo di ottenere con l'esame approfondito dei risultati sperimentali di altri osservatori e con più completi dati astronomici.

\section{RIASSUNTO}

Tengono presentati alcuni risultati delle osservazioni ionosferiche of fettuate in Roma durante l'eclisse di sole del 15 Febbraio 1961. Il comportamento della ionosfera lascerebbe supporre che la superficie ionizzante del sole sia alquanto maggiore di quella del disco visibile.

\section{$S U M M A R Y$}

Some results of the ionosphere observations made in Rom during the solar eclipse of February 15th 1961 are presented. The behaviour of the ionosphere leads to suppose that the ionizing surface of the sun is larger that the visible disk. 